USC-91/HEP-B5

October 1991

\title{
GENERALIZED DUALITY AND SINGULAR STRINGS IN HIGHER DIMENSIONS *
}

\author{
I. BARS and K. SFETSOS \\ Physics Department \\ University of Southern California \\ Los Angeles, CA 90089-0484, USA
}

\begin{abstract}
Deformations of gauged WZW actions are constructed for any pair $(G, H)$ by taking different embeddings of the gauge group $H \subset G$ as it acts on the left and right of the group element $g$. This leads to models that are dual to each other, generalizing the axial/vector duality of the two dimensional black hole manifold. The classical equations are completely solved for any pair $(G, H)$ and in particular for the anti de Sitter string based on $S O(d-1,2) / S O(d-1,1)$ for which the normal modes are determined. Duality is demonstrated for models that have the same set of normal modes. Concentrating on $S O(2,2) / S O(2,1)$, the metric and dilaton fields of the $d=3$ string as well as some of the dual generalizations are obtained. They have curvature singularities and represent new singular solutions of Einstein's general relativity in three dimensions.
\end{abstract}

* Research supported in part by the U.S. Department of Energy, under Grant No. DE-FG0384ER-40168 


\section{Introduction}

In order to study the behaviour of string propagation and interaction in curved space and time anti-de-Sitter (ADS) non-compact current algebra coset models $S O(d-$ $1,2) / S O(d-1,1)$ were introduced as exact conformal theories [1]. Using algebraic arguments as well as the equivalence of cosets to gauged Wess-Zumino-Witten models [2], it was argued in [1] that these theories contain only one time dimension and $d-1$ space dimensions for $d=2,3,4, \cdots 26$ (for the supersymmetric versions $d=2,3, \cdots, 10$ ). The original motivation for these models was to try to learn about the quantum properties of strings in non-trivial space-times (see also [3] ) by using the techniques of non-compact current algebra [4] [5]. With Witten's interpretation [6] of the $d=2$ model $S O(2,1) / S O(1,1)$ as a string propagating in a black hole background in two space-time dimensions, it has become apparent that these models may be able to shed some new light on gravitational singularities [7]. It is also possible to give a cosmological interpretation to the two dimensional solution [8] [9]. These interpretations are obtained by an essentially classical analysis. The exact $d=2, k=9 / 4$ quantum theory has been developed partially $[1,10-15]$ and not surprizingly it reveals new features.

These developments motivate us to investigate the classical and quantum properties of such models in higher dimensions. The list of all gauged WZW models that yield a single time coordinate was completed [8] by considering the hermitian symmetric spaces (HSS) with the group $G$ non-compact and $H^{\prime}$ the maximally compact subgroup. In this case the subgroup always has a $U(1)$ and one may write $H^{\prime}=H \times U(1)$. For the gauge subgroup in WZW model take $H$. Then the coset string is given by $G / H$ and, because of a neccesarily negative central extension, it describes a string with one time coordinate (associated with the $U(1))$ and $\operatorname{dim}\left(G / H^{\prime}\right)$ space coordinates. An example is $S U(2,1) / S U(2)$. The full list is $S U(n, m) / S U(n) \times S U(m), S O(n, 2) / S O(n), S O^{*}(2 n) / S U(n), S p^{*}(2 n) / S U(n)$, $E_{6}^{*} / S O(10), E_{7}^{*} / E_{6}$. The stars on $S O^{*}, S p^{*}, E^{*}$ imply that the real forms for these groups are taken such that the $G / H$ generators are purely non-compact. Further generalizations include supersymmetric versions [1] [8] and direct products of any of the previous cases with any conformal theory that describes purely space components of strings, such that the total central charge is $c=26$ ( $c=15$ with supersymmetry). Using this later approach in conjuction with the original $2 \mathrm{~d}$ black hole, a 3-dimensional charged black string [16] and super black p-branes [17] have been constructed.

In this paper it will be shown that any gauged WZW model described by a pair $(G, H)$ has generalizations that correspond to different embeddings of the gauge group that acts on 
the left or the right of the group element $g$. Although our main motivation is to study the space-time strings described above, the general remarks below apply to any pair $(G, H)$ for compact as well as non-compact groups or (with appropriate modifications) supergroups. The generalized action is

$$
\begin{aligned}
& S=S_{0}(g)-\frac{k}{4 \pi} \int_{M} d^{2} \sigma \operatorname{Tr}\left(A_{-} \partial_{+} g g^{-1}-\tilde{A}_{+} g^{-1} \partial_{-} g+A_{-} g \tilde{A}_{+} g^{-1}-A_{-} A_{+}\right) \\
& S_{0}=\frac{k}{8 \pi} \int_{M} d^{2} \sigma \operatorname{Tr}\left(g^{-1} \partial_{+} g g^{-1} \partial_{-} g\right)-\frac{k}{24 \pi} \int_{B} \operatorname{Tr}\left(g^{-1} d g g^{-1} d g g^{-1} d g\right)
\end{aligned}
$$

where $g\left(\sigma^{+}, \sigma^{-}\right)$is a group element and $A_{ \pm}\left(\sigma^{+}, \sigma^{-}\right)$are the gauge fields associated with $H$. Furthermore, $\tilde{A}_{ \pm}$and $A_{ \pm}$are related by

$$
A_{ \pm}=t_{a} A_{ \pm}^{a}, \quad \tilde{A}_{ \pm}=\tilde{t}_{a} A_{ \pm}^{a},
$$

where $t_{a}, \tilde{t}_{a}$ are matrix representations of the Lie algebra of $H$ embedded in a representation of $G$ 国. The gauge invariance and conditions on $\tilde{t}_{a}$ will be given in section 3 .

In section 2 we will solve generally the classical equations of the standard gauged WZW model (with $\tilde{A}_{ \pm}=A_{ \pm}$) and in particular give explicitly the full solution of the $d$ dimensional ADS model. In section 3 this solution is related to the classical solution of the generalized action (1.1). An interesting aspect of the $2 \mathrm{~d}$ black hole is that it has the property of duality that maps very different regions of the manifold into each other (analog of $R \rightarrow 1 / R$ duality) [18] [10] [11] [19] [20] [8]. One wonders whether this is special to $S O(2,1) / S O(1,1)$ because of the anomaly free choices of vector or axial gauging

1 The sign of $k$ has been changed compared to the compact case so that $k>0$ for $c=26$ and simultaneosly the signature of space-time has the correct sign [5] [1] . Furthermore, in the path integral that defines the quantum theory, the last cubic term in $S_{0}$ is uniquely defined only when the third homotopy group of $\mathrm{G}$ vanishes or is the set of integers, $\pi_{3}(G)=0$ or $Z$ (see Witten in ref.2). If $\pi_{3}=0$ then $k$ can be any real number, while for $\pi_{3}=Z k$ must be an integer. For a non-compact group $G$ the maximal compact subgroup $H_{\max }$ determines $\pi_{3}(G)=\pi_{3}\left(H_{\max }\right)$. If $H_{\max }$ has any non-Abelian part then $\pi_{3}\left(H_{\max }\right)=Z$, and if it is purely Abelian $\pi_{3}\left(H_{\max }\right)=0$. Therefore for all $d \geq 4$ ADS models and all HSS models one must take $k=$ integer. Accordingly, in order to obtain $c=26$ one may be forced to consider direct product type models for some of the cases mentioned. One notable case, which is not a direct product is the $d=4$ supersymmetric ADS model for which $c=15$ when $k=5$ [1]. Another one is the $d=5 S U(2,1) / S U(2)$ bosonic model which gives $c=26$ for $k=4$ [8] . 
of the $S O(1,1)$. In section 3 it is shown how, under special circumstances, the new action generalizes the duality concept to all gauged WZW models (compact or non-compact). This duality is applicable even with non-Abelian gauge subgroups. After these general observations, in section 4 we concentrate on the $d=3$ ADS string and derive the classical gravitational metric and dilaton fields. The dually related metrics are also discussed. These metrics are both time and space dependent and have curvature singularities. The 3d space-time manifolds described by these metrics provide new and unfamiliar singular solutions of Einstein's equations.

\section{Classical Solution}

The equations of motion that follow from (1.1), when $\tilde{A}_{ \pm}=A_{ \pm}$, are

$$
\left(D_{+} g g^{-1}\right)_{H}=\left(g^{-1} D_{-} g\right)_{H}=0, \quad F_{+-}=0, \quad D_{-}\left(D_{+} g g^{-1}\right)_{G / H}=0
$$

where $D_{ \pm} g=\partial_{ \pm} g-\left[A_{ \pm}, g\right]$ and $F_{+-}=\partial_{+} A_{-}-\partial_{-} A_{+}-\left[A_{+}, A_{-}\right]$. The subscripts $H, G / H$ imply that the matrices that represent the quantities in parantheses have to be restricted to the $H$-subspace or $G / H$-subspace. $F_{+-}=0$ requires a pure gauge field $A_{ \pm}=-\tilde{h}^{-1} \partial_{ \pm} \tilde{h}$ (locally). Choosing the gauge in which $\tilde{h}=1$ one finds $A_{ \pm}=0$. In this gauge a global $H$ symmetry remains and (2.1) become

$$
\left(\partial_{+} g g^{-1}\right)_{H}=\left(g^{-1} \partial_{-} g\right)_{H}=0, \quad \partial_{-}\left(\partial_{+} g g^{-1}\right)=0
$$

where the $G / H$ subscript in the last equation is omitted since by using the first equation the same result is achieved. The last equation is now easily solved since it is the same as the ungauged WZW model

$$
g\left(\sigma^{+}, \sigma^{-}\right)=g_{L}\left(\sigma^{+}\right) g_{R}^{-1}\left(\sigma^{-}\right)
$$

where $g_{L}, g_{R}$ are group elements. From (2.2) they must satisfy the decoupled equations

$$
\left(\partial_{+} g_{L} g_{L}^{-1}\right)_{H}=0, \quad\left(\partial_{-} g_{R} g_{R}^{-1}\right)_{H}=0
$$

To proceed further it is useful to parametrize the group elements as a product $g_{L}=h_{L} t_{L}$, $g_{R}=h_{R} t_{R}$ where $h_{L}, h_{R} \in H$ and $t_{L}, t_{R} \in(G / H)$. Then the equations take the form

$$
h_{L}^{-1} \partial_{+} h_{L}+\left(\partial_{+} t_{L} t_{L}^{-1}\right)_{H}=0, \quad h_{R}^{-1} \partial_{-} h_{R}+\left(\partial_{-} t_{R} t_{R}^{-1}\right)_{H}=0
$$


where the $H$ projection needs to be applied only to the second term. These equations are easily integrated

$$
h_{L}\left(\sigma^{+}\right)=P\left[e^{-\int^{\sigma^{+}}\left(\partial_{+} t_{L} t_{L}^{-1}\right)_{H}}\right], \quad h_{R}\left(\sigma^{-}\right)=P\left[e^{-\int^{\sigma^{-}}\left(\partial_{-} t_{R} t_{R}^{-1}\right)_{H}}\right]
$$

where $P$ stands for path ordering. Thus the full solution for a closed string is obtained by considering all coset elements $t_{L}\left(\sigma^{+}\right), t_{R}\left(\sigma^{-}\right)$that are periodic in $\sigma^{ \pm}$(up to possible isometries). The only restrictions on $t_{L}, t_{R}$ come from conformal invariance in the form of vanishing stress tensor $T_{L}, T_{R}$ for left and right movers. Since with (2.6) the subgroup currents are identically zero, the stress tensor is constructed from only the coset currents $\left(\partial_{+} g_{L} g_{L}^{-1}\right)_{G / H}$ and $\left(\partial_{-} g_{R} g_{R}^{-1}\right)_{G / H}$. After some simplification one gets

$$
T_{L}=\frac{1}{2} \operatorname{tr}\left[\left(\left(\partial_{+} t_{L} t_{L}^{-1}\right)_{G / H}\right)^{2}\right]=0, \quad T_{R}=\frac{1}{2} \operatorname{tr}\left[\left(\left(\partial_{-} t_{R} t_{R}^{-1}\right)_{G / H}\right)^{2}\right]=0
$$

There remains to parametrize the coset in some convenient way that allows the solution of these equations.

Up to this point the formalism is general. We now specialize to the ADS model in $d$ dimensions $S O(d-2,2) / S O(d-1,1)$ and parametrize the coset as in [10]

$$
t=\left(\begin{array}{cc}
b & -b X^{\nu} \\
b X_{\mu} & \left(\eta_{\mu}^{\nu}+a b X_{\mu} X^{\nu}\right)
\end{array}\right), \quad \partial t t^{-1}=\left(\begin{array}{cc}
0 & -J^{\nu} \\
J_{\mu} & a b \eta_{\mu \lambda} X^{[\lambda} \partial X^{\nu]}
\end{array}\right)
$$

where $b=\epsilon\left(1+X^{2}\right)^{-\frac{1}{2}}, a=\left(1-b^{-1}\right) / X^{2}, J^{\mu}=b\left(\partial X^{\mu}+X^{\mu} a b X \cdot \partial X\right), \epsilon= \pm 1$ and $\eta_{\mu \nu}=\operatorname{diag}(1,-1, \ldots-1)$ is the Minkowski metric (when $\epsilon=-1$ the group element is not continuously connected to the identity; this will play a role in duality). However, unlike [10] which uses $X^{\mu}\left(\sigma^{+}, \sigma^{-}\right)$, we insert in these expressions $X_{L}^{\mu}\left(\sigma^{+}\right)$or $X_{R}^{\mu}\left(\sigma^{-}\right)$in order to obtain $t_{L}, t_{R}$. The constraints (2.7) take the form $J_{L}^{2}=0=J_{R}^{2}$ which contain non-linear terms and still are difficult to solve. To find more convenient expressions it is useful to make change of coordinates

$$
\left(h_{L}\right)_{\nu}^{\mu} X_{L}^{\nu} b_{L}=V_{L}^{\mu}, \quad\left(h_{R}\right)_{\nu}^{\mu} X_{R}^{\nu} b_{R}=V_{R}^{\mu}
$$

where $h_{L}, h_{R}$ are Lorentz transformations whose $X_{L}, X_{R}$ dependence are determined through (2.5) and (2.8) . After using these equations one obtains the form $h_{\nu}^{\mu} J^{\nu} b=\partial V^{\mu}$ for both $L, R$ sectors. In this way the classical constraints simplify to

$$
\left(\partial_{+} V_{L}\right)^{2}=0, \quad\left(\partial_{-} V_{R}\right)^{2}=0
$$


up to overall factors of $b^{-2}$. These are exactly analogous to the flat string Virasoro constraints and can be solved with identical methods (e.g. by choosing a light-cone gauge for $\left.V^{\mu}\right)$. Furthermore, the solutions (2.6) can be given directly in terms of $V_{L}, V_{R}$ by using (2.5) (2.8) and (2.9) and rewriting

$$
\left(\partial h_{L} h_{L}^{-1}\right)^{\mu \nu}=\frac{V_{L}^{[\mu} \partial V_{L}^{\nu]}}{b_{L}\left(1+b_{L}\right)}
$$

where $b_{L}=\epsilon\left(1-V_{L}^{2}\right)^{\frac{1}{2}}=\epsilon\left(1+X_{L}^{2}\right)^{-\frac{1}{2}}$ and similarly for the $\mathrm{R}$ sector. These equations are solved as path ordered integrals as in (2.6). Thus, in terms of $V_{L}, V_{R}$ the group elements $g_{L}, g_{R}$ take the form

$$
g_{L}=\left(\begin{array}{cc}
b_{L} & -\left(V_{L} h_{L}\right)^{\nu} \\
V_{L \mu} & {\left[\left(h_{L}\right)_{\mu}^{\nu}-\frac{V_{L \mu}\left(V_{L} h_{L}\right)^{\nu}}{1+b_{L}}\right]}
\end{array}\right)
$$

and similarly for $g_{R}$.

It is interesting to point out that this provides a solution to another non-linear equation. In [10] we parametrized $g=h t$ with $t$ given in (2.8). Now $h$ and $t$ depend on both $\sigma^{+}, \sigma^{-}$through $X^{\mu}\left(\sigma^{+}, \sigma^{-}\right)$. It was shown in [10] that the classical equations for $X^{\mu}$ reduce to

$$
\partial_{+}\left[\sqrt{1+X^{2}} \partial_{-}\left(\frac{X^{\mu}}{\sqrt{1+X^{2}}}\right)\right]=0 .
$$

This equation can now be solved for any $d$ by comparing the matrix elements of $g=h t=$ $g_{L} g_{R}^{-1}$ and reading off $X^{\mu}$

$$
X^{\mu}=\frac{1}{b_{L} b_{R}+V_{L} h_{L} h_{R}^{-1} V_{R}}\left[\left(V_{L} h_{L} h_{R}^{-1}\right)^{\mu}-b_{L} V_{R}^{\mu}-\frac{V_{L} h_{L} h_{R}^{-1} V_{R}}{1+b_{R}} V_{R}^{\mu}\right] .
$$

Using (2.11) it can be checked that this indeed solves (2.13) for any $V_{L}^{\mu}, V_{R}^{\mu}$. The expression for $h\left(\sigma^{+}, \sigma^{-}\right)$can similarly be read off by comparing matrix elements of the two parametrizations. It will not be given here because of its lengthy appearance.

The constraints 2.10) may further be written in the usual Virasoro form $L_{n}=0=\tilde{L}_{n}$ by introducing normal modes

$$
\partial V_{L}^{\mu}=\sum \alpha_{n}^{\mu} e^{i n \sigma^{+}}, \quad \partial V_{R}^{\mu}=\sum \tilde{\alpha}_{n}^{\mu} e^{i n \sigma^{-}}
$$

and writing the usual expressions for $L_{n}, \tilde{L}_{n}$. In a light-cone gauge one can take $\alpha_{n}^{+}=$ $\tilde{\alpha}_{n}^{+}=0$ for $n \neq 0$ and solve for $\alpha_{n}^{-}, \tilde{\alpha}_{n}^{-}$in terms of the transverse degrees of freedom and $\alpha_{0}^{+}, \tilde{\alpha}_{0}^{-}$as in the flat string. We have thus completely solved the classical ADS strings in 
terms of $\alpha_{n}^{\mu}, \tilde{\alpha}_{n}^{\mu}$ just like the flat string. The normal modes introduced here are probably useful in developing a light-cone or covariant quantum theory as well, but because of the complexity of quantum ordering and gauge fixing we will not consider this problem in the present paper.

\section{Deformed Gauged WZW models and Generalized Duality}

The ungauged WZW action $S_{0}$ in (1.1) is invariant under $G_{L}\left(\sigma^{+}\right) \times G_{R}\left(\sigma^{-}\right)$transformations which depend on either $\sigma^{+}$or $\sigma^{-}$. The gauged WZW action, when $\tilde{A}_{ \pm}=A_{ \pm}$, is invariant under the gauge transformations $g^{\prime}=h^{-1} g h, A_{ \pm}^{\prime}=h^{-1}\left(A_{ \pm}-\partial_{ \pm}\right) h$, where the fully local $h\left(\sigma^{+}, \sigma^{-}\right)$belongs to the diagonal vector subgroup of $G_{L} \times G_{R}$. It is known that one cannot have full gauge invariance with separate gauge groups for the left and right transformations. However we could try to embed the subgroup differently on the left than on the right. Then covariant derivatives of $g$ take the form $D_{ \pm} g=\partial_{ \pm} g-A_{ \pm} g+g \tilde{A}_{ \pm}$where $A_{ \pm}, \tilde{A}_{ \pm}$are related as given in (1.2). To generate gauge invariant equations of motion but with the new covariant derivative we must have the new action (1.1) with the condition that $\operatorname{Tr}\left(A_{+} A_{-}\right)=\operatorname{Tr}\left(\tilde{A}_{+} \tilde{A}_{-}\right)$. The new equations of motion are

$$
\left(D_{+} g g^{-1}\right)_{H}=\left(g^{-1} D_{-} g\right)_{\tilde{H}}=0, \quad F_{+-}=0, \quad D_{-}\left(D_{+} g g^{-1}\right)_{G / H}=0
$$

where one should note the difference in the subscript $\tilde{H}$ as compared to (2.1). This difference means that $\operatorname{Tr}\left(g^{-1} D_{-} g \tilde{t}_{a}\right)=0$ rather than $\operatorname{Tr}\left(g^{-1} D_{-} g t_{a}\right)=0$. Furthermore, the covariant derivatives have a new meaning as pointed out above.

The condition $\operatorname{Tr}\left(A_{+} A_{-}\right)=\operatorname{Tr}\left(\tilde{A}_{+} \tilde{A}_{-}\right)$together with the requirement that $t_{a}, \tilde{t}_{a}$ must have the same commutation rules place the following constraints on the representation

$$
\operatorname{Tr}\left(t_{a}\left[t_{b}, t_{c}\right]\right)=\operatorname{Tr}\left(\tilde{t}_{a}\left[\tilde{t}_{b}, \tilde{t}_{c}\right]\right), \quad \operatorname{Tr}\left(t_{a} t_{b}\right)=\operatorname{Tr}\left(\tilde{t}_{a} \tilde{t}_{b}\right)
$$

Indeed, with these restrictions one finds that the action (1.1) is invariant under the gauge transformations $g^{\prime}=h^{-1} g \tilde{h}, A_{ \pm}^{\prime}=h^{-1}\left(A_{ \pm}-\partial_{ \pm}\right) h$ or equivalently $\tilde{A}_{ \pm}^{\prime}=\tilde{h}^{-1}\left(\tilde{A}_{ \pm}-\partial_{ \pm}\right) \tilde{h}$. Here $h, \tilde{h}$ contain the same gauge parameters but they are represented differently. This means that $h^{-1} \partial_{ \pm} h=j^{a} t_{a}, \tilde{h}^{-1} \partial_{ \pm} \tilde{h}=j^{a} \tilde{t}_{a}$ with the same $j^{a}$, as required to demonstrate the gauge invariance.

What are the solutions to (3.2) apart from the trivial case $\tilde{t}_{a}=t_{a}$ ? It is immediately

clear that for a $U(1)$ subgroup one can take $\tilde{t}= \pm t$ in accordance with the well known 
vector or axial gauging. Therefore, we concetrate on the non-Abelian part of the gauge group. One solution is $\tilde{t}_{a}=g_{0}^{-1} t_{a} g_{0}$ where $g_{0}$ is any global group element (independent of $\sigma^{+}, \sigma^{-}$) in complexified $G_{R}$. Furthermore $g_{0}$ can also include discrete transformations that are not connected to the identity. In addition, note that the transpose representation will be a new candidate for $\tilde{t}_{a}=-\left(t_{a}\right)^{T}$, which can then be conjugated by any element $g_{0}$. Thus, we have the solutions

$$
\tilde{t}_{a}=g_{0}^{-1} t_{a} g_{0}, \quad \tilde{t}_{a}=-g_{0}^{-1}\left(t_{a}\right)^{T} g_{0}
$$

If there are any additional automorphisms of specific Lie algebras that are not obtainable by (3.3) they will provide additional solutions for $\tilde{t}_{a}$ (which may also be conjugated by some $\left.g_{0}\right)$. We recognize that (3.3) generalizes the vector/axial options that were available for $U(1)$.

Some of these options are not independent. For example consider the $2 d$ black hole, with the $S O(2,1)$ generators $J_{0}, J_{1}, J_{2}$, where $J_{0}$ is antisymmetric and $J_{1}, J_{2}$ are symmetric real $3 \times 3$ matrices, and the non-compact $J_{2}$ gauged. The passage from vector to axial gauging may be achieved by either a $g_{0}$ corresponding to a $180^{\circ}$ rotation around $J_{0}$ $\left(\tilde{J}_{2}=-J_{2}\right)$ or by the second choice in $(3.3)$ corresponding to transposition and $g_{0}=1$. The passage from axial to vector may also be done continuously with a $g_{0}=\exp \left(\alpha J_{0}\right)$. Then $\tilde{J}_{2}=\cos \alpha J_{2}+\sin \alpha J_{1}$. With this choice, taking a unitary gauge for $g$ as before $[6,9,10,18,19]$, and inserting it in the new action (1.1) one finds the metric and dilaton

$$
\begin{aligned}
& d s^{2}=d r^{2}-d t^{2} \frac{\cos \alpha \cosh (2 r)+1}{\cos \alpha \cosh (2 r)-1}+d t d r \frac{2 \sin \alpha}{\cos \alpha \cosh (2 r)-1} \\
& \Phi=\ln (\cos \alpha \cosh (2 r)-1)
\end{aligned}
$$

that shows the role of a continuous $\left(\sigma^{+}, \sigma^{-}\right.$independent) parameter in generating deformed metrics. In particular for $\alpha=0, \pi$ we get the previous vector/axial case respectively. For general $\alpha$ the deformed metric (3.4) does not represent a new manifold, as can be seen by finding the Kruskal coordinates and noting that it takes the standard form given by Witten 6 .

The role played by $\alpha=0$ (original action) and $\alpha=\pi$ (dual action) is reproduced by the $\operatorname{sign} \epsilon= \pm 1$ of the previous section (see the expression for $b$ following (2.8) or (2.11) ) in the unitary gauge. Namely, if the group element is connected to the identity continuously $(\epsilon=1)$ one obtains the metric with $\alpha=0$ and if the group element is in a class disconnected from the identity $(\epsilon=-1)$ one gets the metric with $\alpha=\pi$. Therefore, 
the original action describes both metrics provided all group elements are included (as already known for the $2 \mathrm{~d}$ black hole in Kruskal coordinates [6]). On the other hand, if one insists on only group elements connected to the identity, then the dual actions taken together reproduce the full manifold as parametrized by Kruskal coordinates.

Let us explore a bit further the role of $g_{0}$ as deformation parameters versus a duality transformation. Using the invariance of $S_{0}$ under $G_{L} \times G_{R}$ one may rewrite the full action (1.1) as follows (with $\tilde{A}_{+}=g_{0}^{-1} A_{+} g_{0}$ )

$$
S\left(g, A_{ \pm}^{a}, g_{0}\right)=S\left(g g_{0}^{-1}, A_{ \pm}^{a}, 1\right)
$$

This is not a statement of symmetry (since the result is not $S\left(g, A_{ \pm}^{a}, g_{0}=1\right)$ ). In particular if $g$ is a solution of the $g_{0}=1$ action then $g g_{0}^{-1}$ is not a solution of the same action. Instead, it is a solution of another action that differs from the original one by $g_{0}$. However, we may expect that there are some $g_{0}$ for which we get dual theories. As a criterion for duality we require that the space of classical solutions (or normal modes) of the two dual theories be identical (as for the axial/vector case of the $2 \mathrm{~d}$ black hole [10]). Thus we look for special $g_{0}$ for which the classical solution space is the same in the presence or absence of $g_{0}$. Solving equations (3.1) partially $F_{+-}=0$ and choosing the gauge that gives $A_{ \pm}=0$ (see the previous section) one finds that the only difference in the remaining equations is

$$
\operatorname{Tr}\left(g^{-1} \partial_{-} g g_{0}^{-1} t_{a} g_{0}\right)=0 \quad \text { versus } \quad \operatorname{Tr}\left(g^{-1} \partial_{-} g t_{a}\right)=0
$$

Thus the equations span the same set only when $g_{0}^{-1} t_{a} g_{0}$ is some linear combination of the $t_{a}$ and is orthogonal to any generator in $G / H$. For such $g_{0}$, when they are not trivial global rotations embedded in the gauge subgroup $H$, one obtain a duality transformation. One such example is the $\alpha=\pi$ rotation illustrated above.

This classical duality argument applies also to the case involving the transpose representation. In this case the requirement analogous to (3.6) is automatically satisfied since, in an appropriate basis, $-\left(t_{a}\right)^{T}$ differs from $t_{a}$ at most by some minus signs. Therefore for this case we have automatically dual theories. The same set of $g_{0}$ 's as above may also generate additional dual theories. In some instances it is possible to prove that $-\left(t_{a}\right)^{T}=g_{0}^{-1} t_{a} g_{0}$ for some $g_{0}$. This is certainly the case for ADS strings with e.g. $g_{0}=\operatorname{diag}( \pm 1,-1,1,1, \cdots, 1)$ where either sign \pm in the first entry will do the job. However, this is not always the case, for example for $\mathrm{SU}(\mathrm{N})-\left(t_{a}\right)^{T}$ is equivalent to the complex conjugate representation which may not be obtained via a similarity transformation. 
The path integral based on the dual theory with $-\left(t_{a}\right)^{T}$ is not obviously identical to the original one. However, on the basis of the above classical argument, we expect that by rewriting the path integral as in [19 that the duality can be shown. As mentioned in the previous paragraph in some cases $-\left(t_{a}\right)^{T}$ is obtained with a similarity transformation. Let us consider such cases along with the arbitrary $g_{0}$ deformations. In a quantum theory defined by a path integral one integrates over all group elements, not only solutions. Then at first sight there seems to be no difference between $g$ and $g g_{0}^{-1}$ and therefore one might expect that the new action and the old one give equivalent quantum theories. This would have been the simplest proof of quantum duality for the ADS strings (and in particular of the $2 \mathrm{~d}$ black hole) if the integration measure were the Haar measure for $g$. However there is no reason to assume that the measure be invariant under left or right multiplication with arbitrary group elements. After all, since the action is invariant only under the diagonal (or deformed diagonal) subgroup $H$, it is sufficient to require an invariance of the measure only under $H$. In fact the criterion for the correct measure is conformal invariance including the effects of the gauge field measure. As we shall argue in the next section, indeed the correct measure is not the Haar measure. Therefore, to prove duality at the quantum level one needs to rewrite the path integral as in the example provided in [19]. We will postpone this proof to future work.

\section{ADS Strings in 3 dimensions}

We now study the ADS string in 3 dimensions based on $S O(2,2)_{k} / S O(2,1)_{k}$. As pointed out in [1] there is a generalization of this model with two different central extensions in the form $S O(2,1)_{k_{1}} \times S O(2,1)_{k_{2}} / S O(2,1)_{k_{1}+k_{2}}$. The $k_{1} \neq k_{2}$ version was recently examined [21]. We will analyse the $k_{1}=k_{2}$ version in a parametrization that leads to a diagonal metric. Furthermore, we will discuss the duality properties and the new metrics that follow from the new action (1.1).

At first we take $\tilde{A}_{ \pm}=A_{ \pm}$. We parametrize $g=h t$ with $t$ given in (2.8). Furthermore, for $3 d$ the Lorentz transformation $h$ may be constructed from a vector

$$
h_{\mu \nu}=\epsilon^{\prime} \sqrt{1-Y^{2}} \eta_{\mu \nu}+\frac{Y_{\mu} Y_{\nu}}{1+\epsilon^{\prime} \sqrt{1-Y^{2}}}+\epsilon_{\mu \nu \lambda} Y^{\lambda}
$$

where $\epsilon^{\prime}= \pm 1$ as in (2.8) (signifying group elements connected or disconnected from the identity). In terms of this parametrization the Haar measure for $S O(2,2)$ turns out to be

$$
(D g)=\frac{\left(D^{3} X\right)\left(D^{3} Y\right)}{|f(X, Y)|}, \quad f=\left(1+X^{2}\right)^{2} \sqrt{1-Y^{2}}\left(1+\epsilon^{\prime} \sqrt{1-Y^{2}}\right)
$$


where $\left(D^{3} X\right),\left(D^{3} Y\right)$ are the naive Minkowski or Euclidean measure. As pointed out at the end of the last section, one is not required to take the Haar measure since the action is invariant only under the $H$ subgroup. Therefore, one may modify this measure by any Lorentz invariant factor $F\left(X^{2}, Y^{2}, X \cdot Y\right)$. As will be seen later, this will be necessary because of conformal invariance which will fix $F$. The action contains a quadratic term of the form $A_{+}^{a} M_{a b} A_{-}^{b}$. Integrating out the $A_{ \pm}$gives a determinant that modifies the measure in the path integral by a factor

$$
\operatorname{det}(M)^{-1}=\frac{\left(1+X^{2}\right)\left(1+\epsilon^{\prime} \sqrt{1-Y^{2}}\right)}{\left(X^{2} Y^{2}-(X \cdot Y)^{2}\right)} .
$$

As we shall see, this gauge invariant expression will be identified with the dilaton field $C e^{-\Phi}=\operatorname{det}(M)^{-1}$, where $C$ is a constant. To obtain the effective action one solves the first pair of equations in (2.1) and substitutes the solution for $A_{ \pm}$in the action. The gauge part of the action then reduces to

$$
S_{1}=-\frac{k}{4 \pi} \int \operatorname{Tr}\left(A_{-} \partial_{+} g g^{-1}\right)
$$

where $A_{-}$is the solution. It was shown in [10] that the $A_{-}$equation becomes

$$
\left(h^{-1} D_{-} h\right)^{\mu \nu}=a X^{[\mu}\left(D_{-} X\right)^{\nu]}
$$

where $\left(D_{ \pm} X\right)^{\mu}=\partial_{ \pm} X^{\mu}-\left(A_{ \pm}\right)_{\nu}^{\mu} X^{\nu}$ and $\left(D_{ \pm} h\right)_{\mu}^{\nu}=\partial_{ \pm} h_{\mu}^{\nu}-\left[A_{ \pm}, h\right]_{\mu}^{\nu}$. This equation can be solved for $A_{-}$without fixing any gauges. Substitution in (4.4) leads to a non-linear locally Lorentz invariant action written in terms of the two vectors $X, Y$. We have done this, but because of the complexity of the expression we specialize to a gauge fixed form.

Local Lorentz transformations cannot change the sign or magnitude of $X^{2}, Y^{2}$. Therefore the cases of $X^{2}>0, X^{2}<0, X^{2}=0$ ( similarly for $Y^{2}$ ) have to be gauge fixed separately. Fortunately, it is possible to pass from one sign to the other by analytic continuation of the gauge fixed parameters. Therefore, we concentrate on space-like $X^{2}, Y^{2}<0$. By Lorentz transformations these two vectors can be brought to the form

$$
X^{\mu}=\tanh (2 r)(0, \cos \theta, \sin \theta), \quad Y^{\mu}=\epsilon^{\prime} \sinh (2 t)(0,0,1) .
$$

In choosing this form we were inspired by the two dimensional black-hole (specializing to $\theta=0$ yields the two dimensional case with $r, t$ identified with the parameters used in two dimensions). After some tedius algebra we obtain the gauge fixed form of the action (1.1)

$$
S=\frac{k}{\pi} \int d^{2} \sigma \quad G_{\alpha \beta} \partial_{+} \phi^{\alpha} \partial_{-} \phi^{\beta}, \quad \phi^{\alpha}=(r, \theta, t),
$$


where the target space metric $G_{\alpha \beta}$ is given by the line element $d s^{2}=G_{\alpha \beta} d \phi^{\alpha} d \phi^{\beta}$ and the antisymmetric tensor $B_{\alpha \beta}$ is zero with our parametrization. However, the form that emerges for $G_{\alpha \beta}$ depends on the signs $\epsilon, \epsilon^{\prime}$ chosen in (2.8) and (4.1) as follows

$$
\begin{aligned}
& d s^{2}=d r^{2}+\lambda^{2}(r, \epsilon)\left[d \theta+\kappa\left(t, \epsilon^{\prime}\right) \tan \theta d t\right]^{2}-\frac{1}{\lambda^{2}(r, \epsilon) \cos ^{2} \theta} d t^{2} \\
& \lambda^{2}(r, \epsilon)=\frac{\cosh (2 r)-\epsilon}{\cosh (2 r)+\epsilon} \quad \kappa\left(t, \epsilon^{\prime}\right)=\frac{\sinh (2 t)}{\cosh (2 t)-\epsilon^{\prime}}
\end{aligned}
$$

Depending on these signs the various factors simplify to

$$
\lambda^{2}(r, 1)=\tanh ^{2} r, \quad \lambda^{2}(r,-1)=\operatorname{coth}^{2} r, \quad \kappa(t, 1)=\operatorname{coth}(t), \quad \kappa(t,-1)=\tanh (t)
$$

These forms clearly indicate that the various signs $\epsilon, \epsilon^{\prime}$ are closely related to duality transformations. In fact, we find that the effect of $\epsilon^{\prime}$ is reproduced by a dual theory defined with a $g_{0}=(1,-1,-1,1)$ as in section 3 . The effect of $\epsilon$ is also reproduced by a dual theory with $g_{0}=(-1,1,1,-1)$ provided one works in the gauge $X^{\mu}=\tanh (2 r)(0,0,1), Y^{\mu}=\epsilon^{\prime} \sinh (2 t)(0,-\sin \theta, \cos \theta)$ instead of (4.6). Both of these gauge choices lead to the same metric (4.8) in the original theory.

The metric can be put into diagonal form by changing variables

$$
T=\sqrt{\frac{\cosh (2 t)+\epsilon^{\prime}}{2}}, \quad z=\sqrt{\frac{\cosh (2 t)-\epsilon^{\prime}}{2}} \sin \theta
$$

which gives

$$
d s^{2}=d r^{2}+\frac{\lambda^{2}(r, \epsilon) d z^{2}-\lambda^{-2}(r, \epsilon) d T^{2}}{T^{2}-z^{2}-\epsilon^{\prime}}
$$

where $T^{2}-z^{2}-\epsilon^{\prime}=\frac{1}{2}\left(\cosh (2 t)-\epsilon^{\prime}\right) \cos ^{2} \theta \geq 0$.

The dilaton (which should come out from the integration measure) must satisfy the target space Einstein equation $R_{\alpha \beta}=D_{\alpha} D_{\beta} \Phi$ perturbatively in powers of $1 / k$ in order to satisfy conformal invariance [22]. Obviously, this equation is simpler in the new basis $\phi^{\alpha}=(r, z, T)$. In the two dimensional black hole $\Phi=\ln (\operatorname{det} M)+$ const. solves the dilaton equation. Taking this as a guide we try the form (4.3) after using (4.6) and (4.10)

$$
e^{\Phi-\Phi_{0}}=(\operatorname{det} M)=\sinh ^{2}(2 r) \cos ^{2} \theta\left(\cosh (2 t)-\epsilon^{\prime}\right)=2 \sinh ^{2}(2 r)\left(T^{2}-z^{2}-\epsilon^{\prime}\right)
$$

After tedius algebra one finds that indeed (4.12) solves the curvature equation. Also the central charge of the model can be computed (to leading order in powers of $1 / k$ ) using [22]

$$
c=3+\frac{3}{2 k}\left(D_{\alpha} \Phi D^{\alpha} \Phi+D_{\alpha} D^{\alpha} \Phi\right)
$$


We find $c=3+\frac{18}{k}$ in agreement with the expansion of the exact central charge $c=$ $\frac{6 k}{k-4}-\frac{3 k}{k-2}$ in powers of $1 / k$. Next we compute the scalar curvature

$$
R=-\frac{8}{\sinh ^{2}(2 r)}\left[1+\frac{2 \epsilon \cosh (2 r)\left(T^{2}+z^{2}\right)-\epsilon^{\prime}\left(\cosh ^{2}(2 r)+1\right)}{2\left(T^{2}-z^{2}-\epsilon^{\prime}\right)}\right] .
$$

One sees that there are singularities at $r=0$ and at $T^{2}-z^{2}-\epsilon^{\prime}=0$ (equivalently at $\theta= \pm \frac{\pi}{2}, t=0$ for $\epsilon^{\prime}=1$, or $\theta= \pm \frac{\pi}{2}$ for $\epsilon^{\prime}=-1$ ). We believe that this is a new singular solution of Einstein's equations

$$
R_{\alpha \beta}-\frac{1}{2} G_{\alpha \beta} R=T_{\alpha \beta}
$$

The matter energy momentum tensor in this equation does not vanish. Therefore, unlike the two dimensional black hole which corresponds to a vacuum solution, in the present case there is non-trivial matter.

As mentioned earlier, by chosing the gauge (4.6) we have so far concetrated on the space-like regions $-1<X^{2}<0, Y^{2}<0$. Note that $1+X^{2}>0$ and $1-Y^{2}>0$ must always be satisfied for well defined $S O(2,2)$ group elements in (2.8) and (4.1). The remaining regions can be obtained either by choosing appropriate gauges or by simply doing analytic continuation in the variables $r, t, \theta$ while making sure that one remains within $S O(2,2)$ (in a transformed basis). Thus, for $-1<X^{2}<0,0<Y^{2}<1$ substitute $(r, t, \theta) \rightarrow(r, i \theta$, it); for $0<X^{2}, 0<Y^{2}<1$ take $(r, t, \theta) \rightarrow(i t, i \theta, i r)$ and finally for $0<X^{2}, Y^{2}<0$ replace $(r, t, \theta) \rightarrow(i t, r, i \psi)$ where $\psi$ is non-compact. The metrics obtained from (4.8) through these substitutions all have a single time coordinate designated by $t$. However, due to analytic continuation, not every patch has the same structure of curvature singularities. It is also understood that the metrics for coset theories based on the groups $S O(3,1)$ and $S O(4)$ follow by appropriate analytic continuations of our expressions. Curvature singularities are seen to occur also for these cases.

The analytically continued metrics together with the duality transformed metrics give various patches of the complete space-time manifold (as for the $2 d$ black hole). The shape of the manifold is clearly complicated. Consider the patch given by (4.8) . At constant time $d t=0$ it describes the shape of a semi-infinite cigar for $\epsilon=1$ or a trumpet for $\epsilon=-1$ (like the $2 \mathrm{~d}$ Euclidean black hole metric). However, this shape does not remain static and it evolves with time. It rotates at a rate which is both $t$ and $\theta$ dependent. The meaning of the singularities are presently unclear. To further interpret this manifold one needs to find its global properties by studying the geodesics of light rays, finding the geodesically complete 
metric and writing it in terms of global coordinates (analog of Kruskal coordinates). This remains for future work.

We now turn to an analysis of the measure. The gauge fixing $\left(X^{0}, Y^{0}, Y^{1}=0\right)$ introduces a Faddeev-Popov determinant in the path integral measure which may be computed from the Lorentz transformation properties

$$
\left(\begin{array}{l}
\delta Y^{0} \\
\delta Y^{1} \\
\delta X^{0}
\end{array}\right)=\left(\begin{array}{ccc}
0 & Y_{2} & 0 \\
-Y_{2} & 0 & 0 \\
0 & X_{2} & -X_{1}
\end{array}\right)\left(\begin{array}{l}
\omega_{12} \\
\omega_{02} \\
\omega_{10}
\end{array}\right)
$$

where $\omega_{\mu \nu}$ are the infinitesimal Lorentz parameters. Thus, the Faddeev-Popov determinant is

$$
\Delta=X^{1}\left(Y_{2}\right)^{2}
$$

The remaining integrations are $\left(D X^{1}\right)\left(D X^{2}\right)\left(D Y^{2}\right)=(D r)(D t)(D \theta) J$, where $J$ is the Jacobian due to the change of variables $\left(X^{1}, X^{2}, Y^{2}\right) \rightarrow(r, \theta, t)$ given in 4.6)

$$
J=\left|\frac{\sinh (2 r) \cosh (2 t)}{\cosh ^{3}(2 r)}\right|
$$

Putting all the factors together the integration measure takes the form

$$
(D r)(D t)(D \theta) J \Delta \operatorname{det}(M)^{-1} f^{-1} F
$$

where all the factors, except F, are computed above. It is interesting to observe that the known factors combine to give $D^{3} \phi \sqrt{-G} F$

$$
J \Delta(\operatorname{det} M)^{-1} f^{-1}=\sqrt{-G}=\frac{1}{|\cos \theta|} .
$$

A similar relation holds for the two dimensional black hole as well [19], so maybe one can prove a theorem for any gauged WZW model. Assuming that this relation is general, we give a nice prescription for computing the dilaton without directly evaluating the determinant $\operatorname{det} M$. Namely,

$$
e^{-\Phi}=(\operatorname{det} M)^{-1}=\sqrt{-G} J^{-1} \Delta^{-1} f
$$

This can serve either as a calculational tool or as a check. Finally we are in a position to determine F. Since the dilaton must be identified by exponentiating the entire measure $\sqrt{-G} F$, one must choose

$$
F=J^{-1} \Delta^{-1} f=\frac{e^{-\Phi}}{\sqrt{-G}}
$$


For consistency with Lorentz invariance of the original measure this must be a function of only dot products involving $X^{\mu}, Y^{\mu}$. This is indeed the case since $\exp (-\Phi)$ is written in terms of dot products in (4.3) and $(-G)^{-\frac{1}{2}}=\cos \theta=\left[1-(X \cdot Y)^{2} / X^{2} Y^{2}\right]^{\frac{1}{2}}$. A non-trivial $F$ is necessary for the $2 d$ black hole as well. This factor in the measure seems to have been neglected in previous path integral discussions of gauged WZW models.

We hope to extend these results to the four dimensional case and to investigate the quantum theory.

\section{Note Added}

At the time of preparation of our paper we received a manuscript by Fradkin and Linetsky [23] which overlaps with some of our results. In particular there is agreement with some patches of the three dimensional metric and dilaton even though the parametrization of the group element and the details of the calculations are quite different. 


\section{References}

[1] I. Bars and D. Nemeschansky, Nucl. Phys. B348 (1991) 89.

[2] E. Witten, Nucl. Phys. B223 (1983) 422. ;

K. Bardakci, E. Rabinovici and B. Saering, Nucl. Phys. B301 (1988) 151. ;

H.J. Schnitzer, Nucl. Phys. B324 (1989) 412. ;

D. Karabali, Q-Han Park, H.J. Schnitzer and Z. Yang, Phys. Lett. B216 (1989) 307. ;

D. Karabali and H.J. Schnitzer, Nucl. Phys. B329 (1990) 649. ;

K. Gawedzki and A. Kupiainen, Nucl. Phys. B320 (1989) 625.

[3] J. Balog, L. O’Raifeartaigh, P. Forgác and A. Wipf, Nucl. Phys. B325 (1989) 225.;

P.M.S. Petropoulos, Phys. Lett. B326 (1990) 151. ;

S. Hwang, Nucl. Phys. B354 (1991) 100.

[4] L. Dixon, J. Lykken, and M. Peskin, Nucl. Phys. B325 (1989) 329.

[5] I. Bars, Nucl. Phys. B334 (1990) 125.

[6] E. Witten, Phys. Rev. D44 (1991) 314.

[7] G. Mandal, A.M. Sengupta and S.R. Wadia, "Classical Solutions of $2 d$ String Theory", IASSNS-HEP-91/14.

[8] I. Bars, "Curved Space-Time Strings and Black Holes", USC-91/HEP-B4.

[9] A.A. Tseytlin and C. Vafa, "Elements of String Cosmology", HUTP-91/A49

[10] I. Bars, "String Propagation on Black Holes", USC-91/HEP-B3.

[11] R. Dijkgraaf, H. Verlinde and E. Verlinde, "String Propagation in a Black Hole Background", PUPT-1252 (1991)

[12] M. Bershadsky and D. Kutasov, "Comment on Gauged WZW Theory", HUTP91/A024.

[13] K. Sfetsos, "Degeneracy of String States in 2-d Black Hole and a New Derivation of SU $(1,1)$ Parafermion Characters", USC-91/HEP-S1.

[14] J. Distler and P. Nelson, "New Discrete States of Strings Near a Black Hole, UPR$0462 \mathrm{~T}$ or PUPT-1262.

[15] I.Bachas and E. Kiritsis, "Beyond the Large N limit: Non-linear $W_{\infty}$ Symmetry of the $S L(2, R) / U(1)$ Coset Model", LBL-31213.

[16] J. Horne and G. Horowitz, "Exact Black String Solutions in Three Dimensions", UCSBTH-91-39.

[17] S. Giddings and A. Strominger, " Exact Fivebranes in Critical String Theory ", UCSBTH-91-35.

[18] A. Giveon, "Target Space Duality and Stringy Black Holes", LBL- 30671

[19] E. Kiritsis, "Duality in Gauged WZW Models", LBL-30747.

[20] A.A. Tseytlin, "Duality and the Dilaton", JHU-TIPAC-91008.

[21] Crescimano, "Geometry and Duality of a Non-Abelian Coset Model", LBL-30947.

[22] C.G. Callan, D. Friedan, E.J. Martinec and M. Perry, Nucl. Phys. B262 (1985) 593.

[23] E.S. Fradkin and V.Ya. Linetsky, "On Space-Time Interpretation of the Coset Models in $D<26$ String Theory", HUTP-91/A044 